\title{
Decision Support Systems to Promote Health and Well-being of People during their Working Age: the Case of the WorkingAge EU Project*
}

\author{
Rosa Maria Resende de Almeida ${ }^{5[0000-0003-3721-7214]}$, Adriana Grau \\ Aberturas $^{5}$, Yolanda Bueno Aguado ${ }^{5}$, Maurizio Atzori ${ }^{10000-0001-6112-7310]}$, \\ Alessandro Barenghi ${ }^{3[0000-0003-0840-6358]}$, Gianluca \\ Borghini ${ }^{20000-0001-8560-5671]}$, Carlos Alberto Catalina \\ Ortega ${ }^{5[0000-0003-1788-0596]}$, Sara Comai ${ }^{3[0000-0002-9554-8815]}$, Raquel Losada \\ Durán $^{5}$, Mariagrazia Fugini ${ }^{3}[0000-0002-0692-0153]$, Hatice \\ Gunes ${ }^{4[0000-0003-2407-3012]}$ Basam Musleh Lancis 5[0000-0002-7485-7029], \\ Gerardo Pelosi ${ }^{3}[0000-0002-3812-5429]$, Vincenzo Ronca ${ }^{20000-0002-7174-6331]}$, \\ Licia Sbattella ${ }^{3}$, Roberto Tedesco ${ }^{3[0000-0002-2830-4247]}$, and Tian $\mathrm{Xu}^{4}$ \\ 1 Università di Cagliari - DMI, Cagliari, Italy \\ atzori@unica.it \\ 2 BrainSigns s.r.l., Roma, Italy \\ \{gianluca.borghini, vincenzo.ronca\}@brainsigns.com \\ ${ }^{3}$ Politecnico di MIlano - DEIB, Milano, Italy \\ \{alessandro.barenghi, sara.comai, mariagrazia.fugini, gerardo.pelosi, \\ licia.sbattella, roberto.tedesco\}@polimi.it \\ 4 University of Cambridge - DCST, Cambridge, U.K. \\ \{hatice.gunes, tian.xu\}@cl.cam.ac.uk \\ 5 Instituto Tecnológico de Castilla y León, Burgos, Spain \\ \{carlos.catalina, basam.musleh\} @itcl.es \\ Fundación INTRAS, Spain \\ \{rra, aga, rld, yba\}@intras.es
}

\begin{abstract}
The WorkingAge project aims at improving the psycho-physical condition of workers, with a special focus on ageing subjects. In this context, a Decision Support System, based on a hybrid data-driven / model-driven approach, and fed with data coming from a plethora of environmental and wearable sensors, provides personalised advises to the worker. In this paper we briefly present the WorkingAge project and architecture, and then focus on the decision-making pipeline that, starting from raw data, generates the advises.
\end{abstract}

Keywords: Occupational Safety $\cdot$ Decision Support Systems for Health at Work · Suggestion of Health Strategies · Occupational Ontology.

\footnotetext{
* This project has received funding from the European Union's Horizon 2020 research and innovation programme, under grant agreement N. 826232.
} 


\section{Introduction}

The increasing longevity of population in industrialized countries is shifting the age distribution of the workforce. However, the aging process can lead to physiological and cognitive changes that can affect the well-being and quality of life of workers. Workplace design and organizational strategy can play a pivotal role in maximizing the comfort and performance of occupants, as well as in promotion healthy habits in working/living environments.

The ongoing EU project WorkingAge (Smart Working environments for all Ages - https://www.workingage.eu/) - WA for short - focuses on these issues and promotes healthy habits in working environments targeting people aged over 45. The aim of the project is to achieve a better understanding of well-being at work and of factors that may inhibit or deteriorate prolonged employment 9 . The Working Age Of Wellbeing (WAOW) tool is under development to provide workers with assistance in their everyday routine in the form of recommendations, risks avoidance and reminders. The WAOW tool monitors the worker's behavior, health data and preferences through continuous data collection using Internet of Things (IoT) devices -sensors, interaction tools, wearable devicesto provide recommendations on working habits, physical activities and social relations. The core of the WAOW tool environment is a Decision Support System (DSS), a reasoning system about the worker conditions and for early detection of possible issues. The DSS supports workers in taking effective and personalized decisions and interventions based on a set of measured indicators.

The paper is organized as follows. Section 2 reports related work; Section 3 provides an overview of the WAOW tool; Section 4 introduces the intervention strategies to improve workers' well being; Section 5 introduces the WAOW Ontology-based data model; Section 6 shows how the DSS provide recommendations to the workers; finally, Section 7 outlines conclusions and future work.

\section{Related Work}

Safety and well-being at work is recognized to be highly related to social, economic, and environmental conditions [19]. These should be enhanced to improve health and well aging [12, in the line of what recommended by the Occupational Health and Safety (OHS) management system standard ${ }^{6}$ described in [6. An interesting overview of problems related to aging and well-being at work and in societal environments is given in 13 .

Working, even at a relatively advanced age, is considered good to maintain physical and mental health [10. However, not all workers benefit of favorable conditions, due to dangerous or stressing work environments. Moreover, ageing and shrinking of work forces [2] worsen the work conditions of many classes of workers, due to staff shortage and lack of expertise in young generations [1]. New working and living conditions are encouraged in many studies and experiences,

\footnotetext{
6 https://www.bsigroup.com/en-GB/Occupational-Health-and-Safety-ISO-45001/
} 
centered around concepts of autonomy and quality of life 4 48, The WAOW tool can be classified as a Self-Management Occupational Safety and Health Supervision System (SMOSHS System) [3. The tool is being designed considering DSS issues, in order to adapt its interface and functions to the user, in the line of what proposed in [15] and considering that the partners involved in experiments apply procedures compliant with GDPR.

Coming to the DSS, in the last years, DSSes have become quite popular in health services, to enhance interaction between patients and their physicians [16], in social care [5, and in many other areas, using technologies which commonly draw on an existing knowledge base of evidence and guidelines to provide logical reasoning-based expert advice. In [21, a DSS is described for workers with disabilities who are more sensitive for stress at work and for the injuries connected with non-adequate workplaces. Based on ontologies, many DSSes have been developed for business decision making, or for cyber-physical systems [17/18, which are very similar to our application area. In fact, these systems tightly integrate software, human, and physical components with the need to satisfy constraints on performance and safety of the monitored activities, exhibiting a high degree of automation for the management of their functionalities and decision making processes. In general, there is a strong need for semantic models of the involved application domains. In such a context, the clinical domain 14 witnesses an increasing demand to develop knowledge-based DSSs employing medical knowledge and expertise coming from different ontologies.

\section{The WAOW Tool}

The main goal of the WA project is designing and developing the WAOW tool. Such tool provides feedback to the employers by means of warnings and advises. Furthermore, the WAOW tool collects information useful to perform changes in the workplace that may lead to an increased level of comfort for the workers and new solutions to promote flexible and sustainable job longevity measures. The WAOW tool is focused on the usage of innovative Human Computer Interaction (HCI) methods, including augmented reality, virtual reality, gesture/voice recognition and gaze tracking. It will measure the user's psychological/emotional/health state, collect data about the environmental conditions of her/his workplace, and will provide advises taking into consideration also gender, ethics and security aspects. The WAOW tool architecture can be divided into three parts (see Fig. 11. Firstly, there are numerous sensors that are deployed in the workplace or worn by the worker in order to acquire all the information (Raw data) required by the system; notice that, another input source is provided by questionnaires administered to the worker by means of the WA App, running on the worker's phone. These pieces of information are then sent via Wi-Fi or Bluetooth to the Edge Cloud (the second part of the architecture) to be processed by means of different algorithms to the end of computing a relatively small set of High-Level information regarding the well-being status of the workers. Finally, High-Level information is sent to the WA App, where it is 


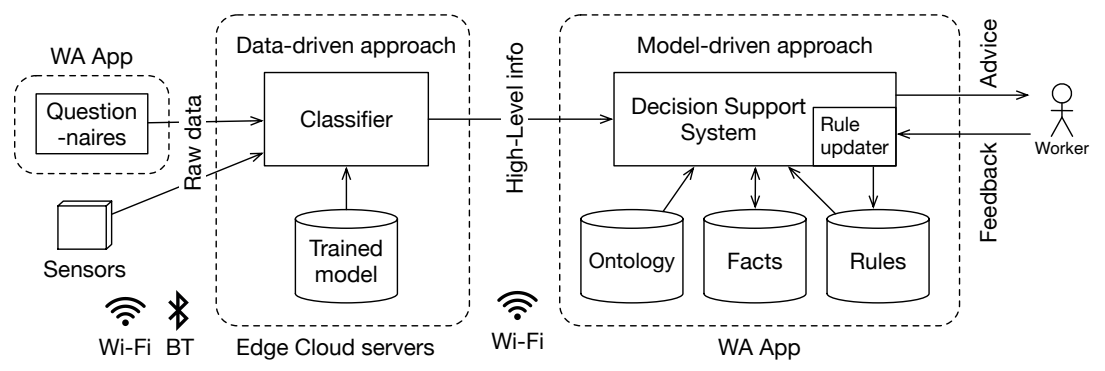

Fig. 1. The WAOW tool decision making pipeline.

stored into the Ontology and processed by the DSS, which generates advises to the worker (see Section 6 for details).

\section{The Intervention Strategy}

The WA project intends to develop an $e$-coach system of interaction to implement an intervention program with workers over 45 years to prolong their work ability and autonomy, reduce strain in different working conditions and promote wellbeing throughout different areas of their lives, promoting an inclusive approach to the health at work. It seeks to increase awareness of workers on aspects such as ergonomic conditions, occupational hazards, stress and its effects on health, providing the worker with the knowledge and skills to reduce negative effects or adopt healthier and safer behaviors.

The WA Intervention Framework is pillared on well-known models, such as the CDC Workplace Health Model, the Plan-Do-Check-Act (PDCA) cycle adopted by the Occupational Safety and Health Administration for the certification process of the OHSAS 18001 management standard, and the Integrated Risk Management (IRM) model, a set of practices and processes supported by a risk-aware culture. It considers the multi-factorial nature of subjective wellbeing and work ability to ensuring a holistic and comprehensive approach to the individual. The WA Intervention Strategy designed so far considers wellness on physical, emotional, intellectual, occupational, social and environmental dimensions. The integrated approach fosters awareness on psycho-social and ergonomic risk prevention measures, seeking to promote attitudinal and behavioral changes towards healthy lifestyles. From this perspective, WA addresses four major areas of intervention: Ergonomics 8 Physical environment (which promotes adequate work postures and encourages healthy work habits), Worker's health and personal characteristics (which encourages the user to follow and/or acquire health promotion and disease prevention strategies), Psycho-social factors (which aims at increasing the self-awareness about cognitive and emotional states of activation or discomfort) and Lifestyle $\&$ Health habits (which promotes the acquisition and maintenance of healthy lifestyle habits). The goal of the WA Interventions 
is not limited to leveraging the power of technology for recommend practices for safety and health but also for general well.being. Taking advantage of the technological concept of WA the proposed intervention approach aims to change the way the workers see and take care of their health, helping to better understand their own health, improving self-awareness of well-being, and changing how workers access to assessment data and advice. For employers, WA provides many opportunities for smarter health and well-being support. In the development of the WA Intervention Framework and Strategies, a multidisciplinary team of expert professionals designed the intervention around the following pillars.

Framework for stress and strain assessment and intervention. Strain is an essential aspect for assessing the effects of work on a given person describing her/his reactions to the set of working conditions. Stress describes the external characteristics of a work situation that influences the working person. These include, for example, physical and organizational working conditions. Within the WAOW tool, there will be different sensors used to measure physical (muscular, skeletal, cardiovascular and somatic) and psychological (mental and emotional) strain, as defined in [20. The analysis of these measures lets the WAOW tool to generate a personalized advice, which aims at improving the worker's condition, based on the strain typology.

WA focuses the design of the intervention in a goal-oriented approach, where coaching is "essentially about helping individuals regulate and direct their interpersonal and interpersonal resources to better attain their goals" [11. Specific, Measurable, Achievable, Relevant Time bound (SMART) goals will be progressively tuned around the user profile, promoting behavioral changes, improving the healthy habits of the worker, taking into account behavioral, environmental and personal factors. Moreover, a specific set of interventions will be developed to meet the different SMART goals along the WA tool usage.

The Recommendation System. The DSS, incorporating the framework depicted above, will generate the appropriate recommendation. Relevant information for the DSS will come from measurements, from SMART goals and from questionnaires (see Section 6). The DSS will propose an advice at any moment, and/or in response to a specific measure, indicative of a possible risk to the user's health. The recommendations are focused on ergonomics, psycho-educational and emotional activation control techniques, and behavioural modification.

\section{The Ontology}

The WA Ontology represents the data model for the High-level information samples generated by Edge Cloud servers. Starting from such pieces of information, the DSS will generate personalized advices to the workers.

The Ontology is represented by means of the OWL2 DL language. In the OWL language, a concept is represented as a class, if it can be seen as a set; the relationships between classes are named object properties; the attributes belonging to a class are named datatype properties; finally, individuals define the 


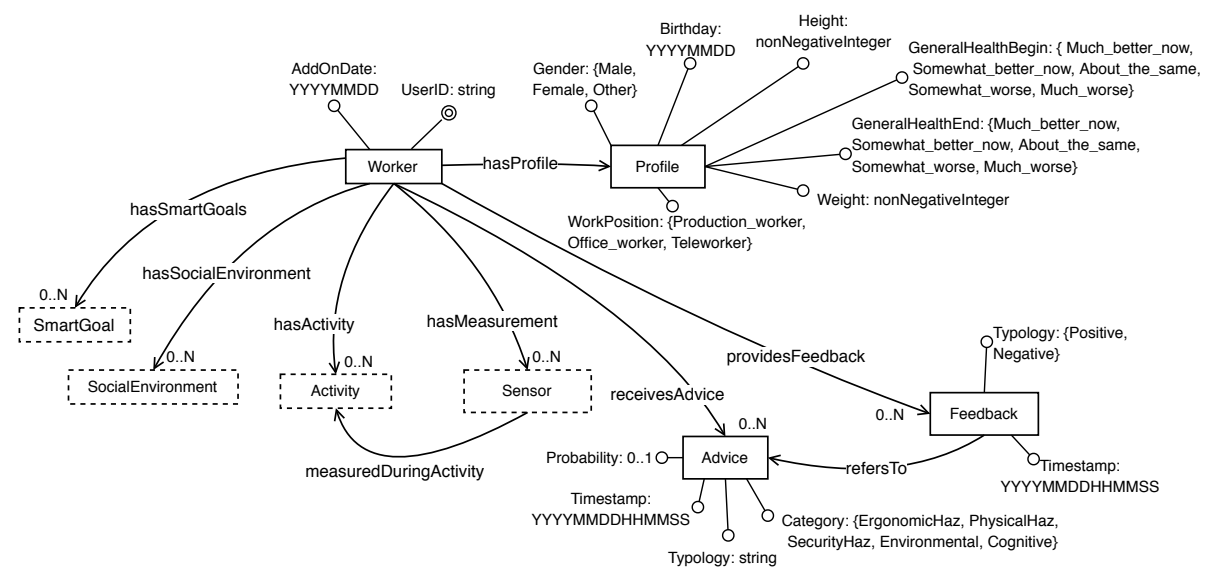

Fig. 2. Description of the worker, and related classes.

instances of a class, i.e., elements of a set. For providing the best privacy, the resulting OWL file is stored on the worker's phone, into the WA App.

In our Ontology each datatype property corresponds to a specific High-level information, and whenever a new High-level information is added to the Ontology, a new individual is created. In the following, we describe the five parts that compose our Ontology, using a simple graphical language, where squares represent classes, arcs show object properties and "pins" depict datatype properties. A dotted square denotes a "link" to a class that is described into other drawings.

Worker. This part is the "core" of the Ontology and describes the profile of the worker, as well as the connections to all the relevant information provided by other parts of the Ontology (see Fig. 2). The Worker class, together with the Profile class, describe the basic characteristics of the worker. Notice that UserID is described as a key for the class Worker, meaning that such a datatype property will be used by the WAOW tool to identify a specific worker. A worker is associated with zero or more advices, and can provide a corresponding feedback. advices are associated with a probability; this is due to the fact that any Highlevel information is, in general, generated by data-driven classifiers. We leverage that characteristic by providing not only the classifier output (which corresponds to a High-level information to be stored into the corresponding datatype property of a specific class) but also the related probability. Thus, the ProbLog engine can provide a probabilistic advice; such probability can be seen as a reliability score associated with the advice. Finally, the worker is associated with several sensor measurements, social environments, activities, and SMART goals. Note that a sensor measurement happens in the context of a given activity; in fact, a worker can be measured at the workplace or during free time.

Sensor. This is the most complex part of the Ontology and describes the High-level information samples calculated from the Edge Cloud servers, about a 


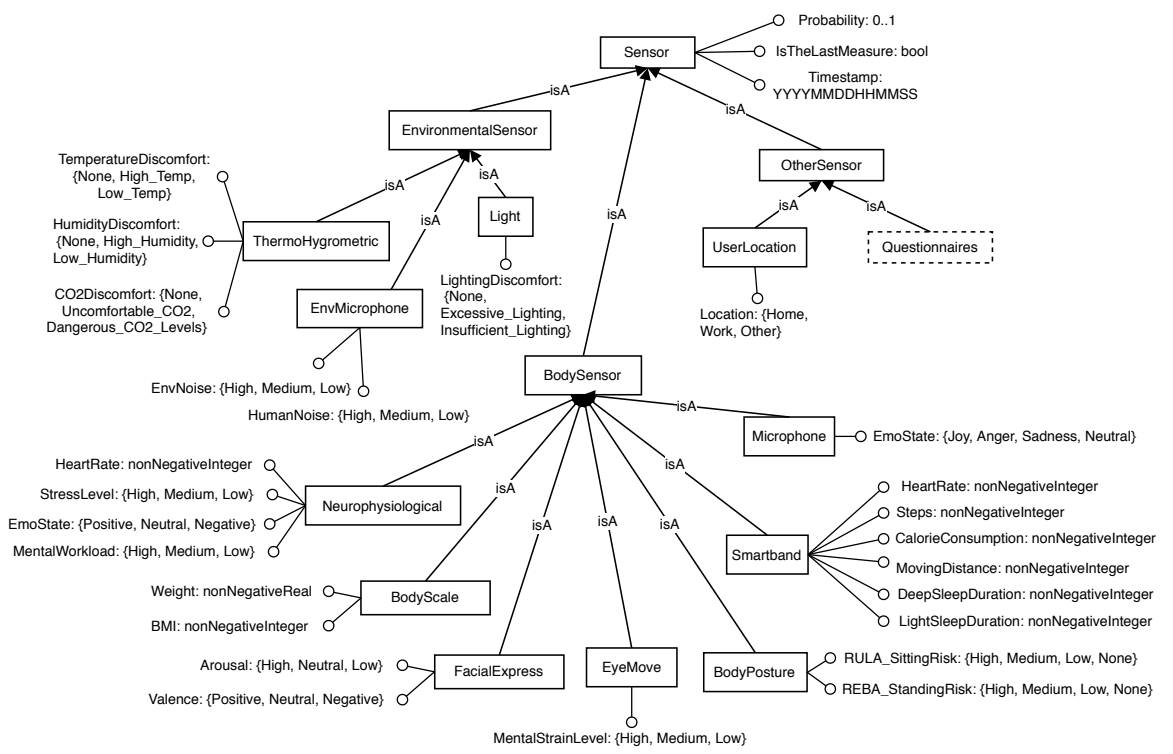

Fig. 3. Information derived from several body and environmental sensors (Questionnaires class omitted).

worker. The taxonomy in Fig. 3 shows the sensor typologies that the WAOW tool leverages for collecting information about the worker and her/his environment. Each measurement is provided with a timestamp and a probability value; over the time, we collect an ordered sequence of High-level information samples, of various typologies, associated with various reliability values (adopting the same interpretation described for advices). The DSS will reason on this sequence. Sensors are subdivided into environmental sensors, body sensors, and other typologies (which include questionnaires and user location). Questionnaires, which will be administered to the worker by means of the WA App, are considered as a special case of sensors, and provide daily, weekly or monthly "measurements".

Activity and social environment. The class named "Activity" describes the relevant characteristics of the activity associated with a worker. Worker's activities are split into two main parts, as Fig. 4 shows: Task and FreeTime. In fact, the WAOW tool will measure workers at the working place, and during their activities at home or in other places. By "social environment" we mean the relevant social characteristics of the working environment as captured by classes in Fig. 5 .

SMART Goals. This part describes the so-called SMART goals about healthy habits that the worker is encouraged to adopt (see Fig. 66). According to the worker's context, a SmartGoal will be set for each individual worker. The Ontology stores the current state of the individual worker as well as the goals that are identified to be met by the worker. These personalized goals can help the work- 


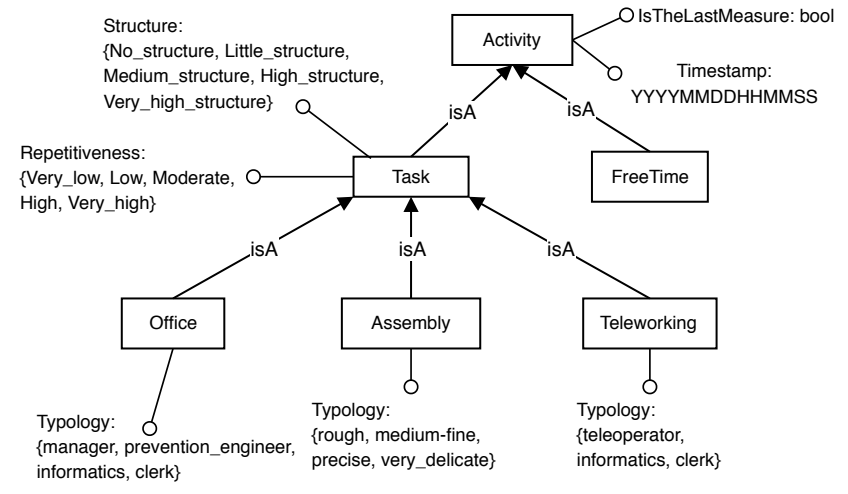

Fig. 4. Information about activities of the worker.

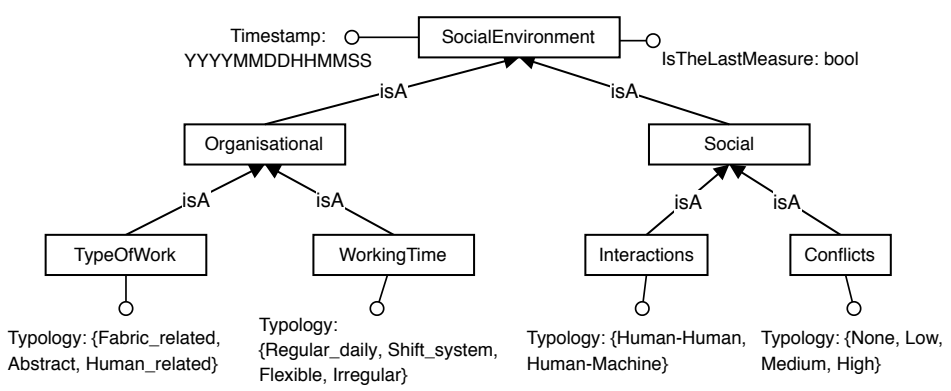

Fig. 5. Information about social characteristics of the working environment.

ers improve their working and living conditions. The Class GoalState shows the worker's progress towards the set SMART goal, which is updated weekly. These goals are mostly related to nutrition, sleep, and social relations, and aim at challenging the workers in terms of behavioural change, and of motivating them to adopt a healthier lifestyle.

\section{The Decision Support System}

One of the central components of the WAOW tool is the DSS; such a system supports the worker while dealing with working activities. The DSS we developed is based on a model-driven approach - in particular, it's a rule-based engine but it is able to work with the results of the Edge Cloud algorithms, which generate a probabilistic output. Thus, the whole "decision-making pipeline", as Fig. 1 shows, is actually based on a hybrid data-driven/model-driven approach. We opted for this design as for the models working on Raw data we found feasible corpora that permitted to leverage data-driven models. For generating advices, however, no feasible corpora existed, and creating our own dataset was not an 


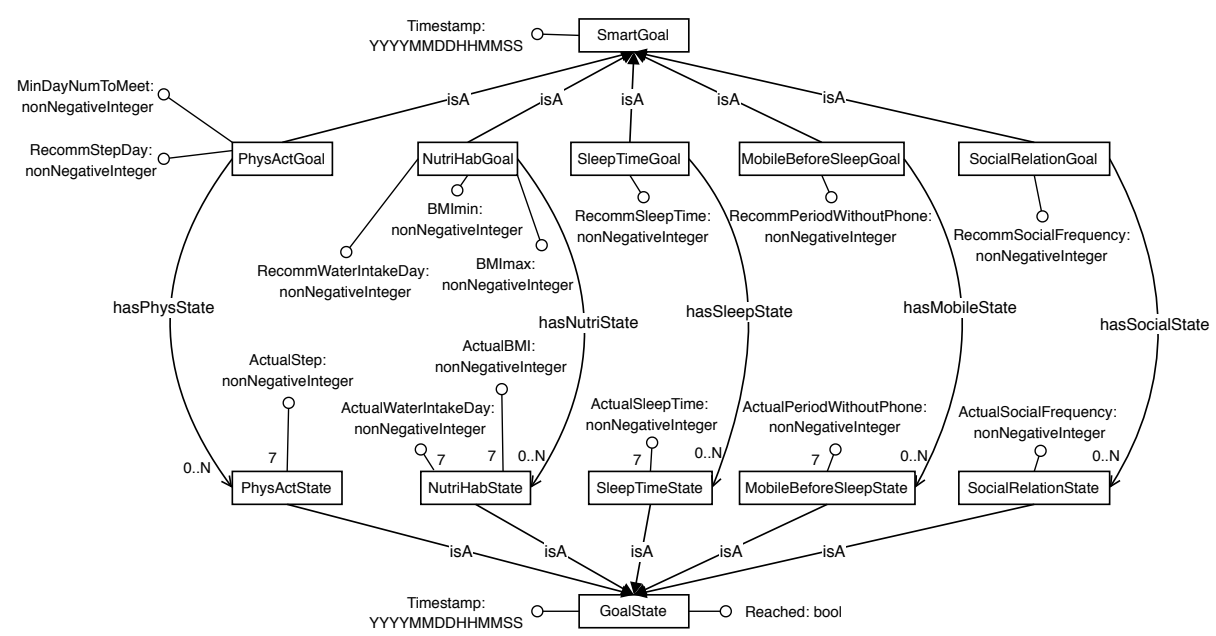

Fig. 6. Information about the SMART goals.

option. Thus, we decided for a model-based approach. Model-driven approaches refer to methods that are able to reason over existing and infer new knowledge based on a given model of the domain at hand. These solutions are often referred to as expert systems. A typical model is often expressed as a set of rules, which describe how to derive new facts, as it happens in well-known declarative programming languages like Prolog. Declarative model-driven approaches are also used for case-based reasoning, to provide insights based on previously occurred instances. Given a model, the computation is performed by the reasoning engine, that applies the model to user-provided data to infer new knowledge regarding the domain.

Research in model-based reasoning focus in various areas, including the core engine optimizations, debugging, expressivity of rules and knowledge representation in general. Recent research included uncertainty in their proposed models, leading to engines able to handle probabilistic rules [7. In the design of the WAOW tool, we determined that uncertainty was needed in order to represent aspects of the model which cannot be predicted in a deterministic way. For instance the confidence that an expert may give to a specific suggestion, or the measure of a sensor, may not be always equal to $100 \%$. This is why we chose ProbLog as the inference engine for the DSS.

ProbLog. ProbLog [7] is a probabilistic logic programming proposed by the DLAI group at the KU Leuven University (Belgium), heavily inspired by Prolog. It reuses the Prolog syntax but allows probabilistic labeling of rules and facts, allowing not only inference of certainly-true facts (according to the usual declarative models), but also derive the level of confidence for that specific inferred fact. An interesting property of ProbLog is that it does not require to specify an exact Bayesian Network, relying instead on a sort of best-effort approximation whenever some nodes and edges of the full causal graph are missing. It can also 
compute marginal probabilities of any number of ground atoms in the presence of evidence, therefore recomputing probabilities whenever new data is provided. This is important since in many real applications, including those in the context of the WA project, many aspects of the causal dependencies cannot be modeled exactly, and so a level of approximation must be handled by the tool. As the original Prolog, ProbLog uses procedural interpretation of Horn clauses, in this case labeled with probabilities. As we show next, we use these probabilistic rules to model scenarios in the context of working environments.

The DSS. As Fig. 1 shows, the DSS is fed by High-Level information generated by the data-driven models running into the Edge Cloud. The Ontology acts as a data model with the aim of describing all the possible worker's physical, cognitive and emotional states.

The DSS leverages the OWL definition of the Ontology to check for correctness and consistency the coming High-level information. Individuals, however, are not described in terms of OWL syntax elements: for best efficiency, they are directly created as probabilities facts, following the ProbLog syntax. The outputs of the DSS will be highly supported by the Interventions platform, described in Section 4. The suggestions and interventions implemented in the WAOW tool are provided by experts. Such suggestions and interventions proposed to the user will be implemented as probabilistic rules by the DSS: the selection is carried out taking into account the probability associated to each worker's state. Such a probability is modulated by the High-Level information provided by each sensor integrated into the WAOW tool and by the feedback provided by each worker toward the interaction with the DSS.

The rules leveraged by the DSS will consider different factors that may affect the well-being of the worker, such as the environment (e.g., loudly, incorrect lighting), wrong behaviour at work (e.g., use of wrong tools or used in a wrong way, posture), worker's habits and state (including out-of-work elements such as food, sleep). The DSS will interact with different entities integrated in the WAOW tool. In particular, the DSS will receive in input the descriptions of the worker's physical and mental states, with the support of the Ontology, and will produce recommendations based on the worker's current state and on her/his history (both stored by the DSS as probabilistic facts). Notice that the worker can provide feedback to the DSS which, in turn, can update the rules to customise them to the worker's preferences. For instance, if a given suggestion in the past (e.g. the use of earmuffs) did not produce an expected benefit (e.g. "decrease in user stress recorded through sensors"), the DSS may then explore other suggestions with the given user (e.g., "take a pause of X minutes").

Action Rules: a simple example for user suggestions. Let's assert, in Prolog, that Jack is a worker and every worker is also a person:

worker (jack).

person $(\mathrm{X})$ :- $\operatorname{worker}(\mathrm{X})$.

The left hand side of the rule is called head, the right hand side is the body. Uppercase $\mathrm{X}$ is a variable. Whenever the body holds, the head holds too. With this 
simple representation, the DSS can automatically infer that Jack is a person, that is, person(jack) holds. In our experiments, we modelled many possible aspects of different working environments, from worker profile -for example, education or activity typology - to time constraints, and even complex behavioural knowledge; as an example, the following rule:

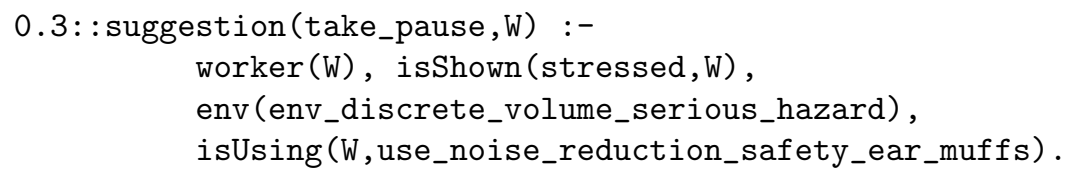

generates the take_pause suggestion whenever a worker in an excessively loud environment is stressed despite using safety ear muffs, giving to the rule a weight of 0.3. The final probability associated to the suggestion takes into account the probability associated to each proposition in the rule body. More than one suggestion could be generated by the DSS. Thus, the associated probability helps in ranking them and select the best one. Moreover, if the selected suggestion is associated with a probability lower than a given threshold, the DSS could simply give up, avoiding to flood the worker with useless advices. Finally, the generated suggestion -for example, take_pause- is translated into human language: "The working area is too noisy and you look tired, it's time to take a pause."

\section{Conclusion}

The paper has described the WA project and tools, aimed at providing smart assistance to elder workers. The paper has focused on the Ontology and the DSS portions of WA, which will generate recommendations about risk avoidance and well-being at work. Experiments about DSS are being conducted via incompany use cases, testing the proposed solutions by collecting sensor data and by improving the learning and smart capabilities of the DSS.

\section{References}

1. Birkel, H.S., et al.: Development of a risk framework for industry 4.0 in the context of sustainability for established manufacturers. Sustainability 11(2), 384 (2019)

2. Calzavara, M., Battini, D., Bogataj, D., Sgarbossa, F., Zennaro, I.: Ageing workforce management in manufacturing systems: state of the art and future research agenda. International Journal of Production Research 58(3), 729-747 (2020)

3. Chen, H., Hou, C., Zhang, L., Li, S.: Comparative study on the strands of research on the governance model of international occupational safety and health issues. Safety science 122 (2020)

4. Cheng, Z., Nielsen, I., Cutler, H.: Perceived job quality, work-life interference and intention to stay. International Journal of Manpower (2019)

5. Cresswell, K., et al.: Investigating the use of data-driven artificial intelligence in computerised decision support systems for health and social care: A systematic review. Health Informatics Journal (2020) 
6. Darabont, D.C., Bejinariu, C., Baciu, C., Bernevig-Sava, M.A.: Modern approaches in integrated management systems of quality, environmental and occupational health and safety. Calitatea 20(S1), 105 (2019)

7. Fierens, D., den Broeck, G.V., Renkens, J., Shterionov, D., B. Gutmann, I.T., Janssens, G., Raedt, L.D.: Inference and learning in probabilistic logic programs using weighted boolean formulas. Theor. Pract. Log. Prog. 15(3), 358-401 (2015)

8. Fitzpatrick, M.D., Moore, T.J.: The mortality effects of retirement: Evidence from social security eligibility at age 62. J. Public Econ. 157, 121-137 (2018)

9. Fugini, M., Barenghi, A., Comai, S., Pelosi, G., Tedesco, R., van Gasteren, M., Catalina, C., Arribas Leal, E., Losada Durán, R., Resende de Almeida, R., Mertens, A., Rick, V.: Workingage: Providing occupational safety through pervasive sensing and data driven behavior modeling. In: Proceedings of the 30th European Safety and Reliability Conference and the 15th Probabilistic Safety Assessment and Management Conference. Research Publishing (2020)

10. Golightley, M., Goemans, R.: Social work and mental health. SAGE Pub. (2020)

11. Grant, A., Stober, D.: Evidence Based Coaching Handbook: Putting Best Practices to Work for Your Clients. Wiley (2006)

12. Grosch, J.W., Scholl, J.C.: Designing age-friendly workplaces: An occupational health perspective. In: Current and Emerging Trends in Aging and Work, pp. 429-451. Springer (2020)

13. Kalteh, H.O., Mortazavi, S.B., Mohammadi, E., Salesi, M.: The relationship between safety culture and safety climate and safety performance: a systematic review. International journal of occupational safety and ergonomics pp. 1-11 (2019)

14. Lodh, N., Sil, J., Bhattacharya, I.: Graph based clinical decision support system using ontological framework. In: International Conference on Computational Intelligence, Communications, and Business Analytics. pp. 137-152. Springer (2017)

15. Mabkhot, M.M., Amri, S.K., Darmoul, S., Al-Samhan, A.M., Elkosantini, S.: An ontology-based multi-criteria decision support system to reconfigure manufacturing systems. IISE Transactions 52(1), 18-42 (2020)

16. Moreira, M., Rodrigues, J., Korotaev, V., Al-Muhtadi, J., Kumar, N.: A comprehensive review on smart decision support systems for health care. IEEE Systems Journal 13(3), 3536-3545 (2019)

17. Pease, S.G., et al.: An interoperable semantic service toolset with domain ontology for automated decision support in the end-of-life domain. Future Generation Computer Systems 112, 848-858 (2020)

18. Petnga, L., Austin, M.: An ontological framework for knowledge modeling and decision support in cyber-physical systems. Adv. Eng. Inf. 30(1), 77-94 (2016)

19. Roughton, J., Crutchfield, N., Waite, M.: Safety culture: An innovative leadership approach. Butterworth-Heinemann (2019)

20. Schlick, C., Bruder, R., Luczak, H.: Arbeitswissenschaft. Springer-Verlag (2018)

21. Vujica Herzog, N., Harih, G.: Decision support system for designing and assigning ergonomic workplaces to workers with disabilities. Ergonomics 63(2), 225-236 (2020) 